\title{
Melanin-Containing Films: Growth from Dopamine Solutions versus Layer-by-Layer Deposition
}

\author{
Falk Bernsmann, ${ }^{[\mathrm{a}]}$ Ovidiu Ersen, ${ }^{[\mathrm{b}]}$ Jean-Claude Voegel ${ }^{[\mathrm{a}, \mathrm{c}]}$ Edward Jan, ${ }^{[\mathrm{d}]}$ Nicholas A. Kotov ${ }^{[\mathrm{d}]}$ \\ and Vincent Ball ${ }^{*[a, c, e]}$
}

Films formed by oxidation of dopamine are of interest for functionalisation of solid-liquid interfaces owing to their versatility. However, the ability to modulate the properties of such films, for example, permeability to ionic species and the absorption coefficient, is urgently needed. Indeed, melanin films produced by oxidation of dopamine absorb strongly over the whole UV/Vis part of the electromagnetic spectrum and are impermeable to anions even for a film thickness as low as a few nanometers. Herein we combine oxidation of dopamine to produce a solution containing dopamine-melanin particles and their alternating deposition with poly(diallyldimethylammonium chloride) to produce films which have nearly the same morphology as pure dopamine-melanin films but are less compact, more transparent and more permeable to ferrocyanide anions.

\section{Introduction}

Surface modification methods are a major research topic in surface science owing to their technological applications such as protection against corrosion, control of wettability ${ }^{[1,2]}$ and modification of adhesion properties, for example, in the field of biomaterials. Popular strategies to modify the functionality of a surface include plasma polymerization, ${ }^{[3]}$ layer-by-layer $(\mathrm{LBL})$ deposition of polymers ${ }^{[4,5]}$ (driven by electrostatic interactions, ${ }^{[6]}$ charge transfer interactions ${ }^{[7]}$ or hydrogen bonding ${ }^{[8]}$ ) and the recently introduced deposition of "poly-dopamine"[9] (which is actually dopamine-melanin ${ }^{[10]}$ ) by oxidation of dopamine with dissolved oxygen.

Surface coatings made from dopamine ${ }^{[9]}$ or norepinephrine $^{[11]}$ solutions can be deposited in a single-step or multistep process and allow for modification of all classes of known materials with a biocompatible material. ${ }^{[12]}$ This material can bind proteins in a covalent manner at physiological $\mathrm{pH}^{[13,14]}$ and absorbs light over the whole UV/Vis spectrum owing to its structure, most probably consisting of polydisperse 5,6-dihydroxyindole aggregates. ${ }^{[15,16]}$

Melanin particles from Sepia officinalis have a hierarchical structure, as revealed by atomic force microscopy. ${ }^{[17]}$ Melanin is fascinating from a fundamental point of view owing to its incompletely explained structure and black colour. The physical principles behind this peculiar colour seem not to be related to light scattering ${ }^{[18]}$ but rather to the overlap of $\pi$-electron conjugated chromophores and inter-chromophoric interactions. ${ }^{[19]}$ Last but not least, melanin is a natural photoprotectant of skin and hair, is believed to have antioxidant properties, to be implicated in Parkinson's disease and to be an electrically conducting material that also exhibits photoconductivity. ${ }^{[20]}$ Melanin coatings ${ }^{[21]}$ may thus be model coatings not only for biological investigations but also for smart surface science.

We recently found that melanin coatings made by oxidation of dopamine at $\mathrm{pH} 8.5$ in the presence of $50 \mathrm{~mm}$ tris(hydroxy- methylaminomethane) became impermeable to ferrocyanide anions at a film thickness of only $7-10 \mathrm{~nm} \cdot{ }^{[10]}$ This is of interest for many applications, but it may also be desirable to have melanin-rich coatings that are highly permeable to small ions to create permselective materials.

The aim of this investigation is to combine the concept of dopamine oxidation and LBL self-assembly to produce films of controllable thickness and permeability. In particular, we compare dopamine-melanin films formed directly by auto-oxidation of dopamine on the substrate (method 1) and (PDADMAC/dopamine-melanin) ${ }_{n}$ LBL films formed by $n$ alternating dipping steps of the substrate in solutions of poly(diallyldimethylammonium chloride), PDADMAC, and preformed dopamine-melanin (method 2). In this case dopamine-melanin is

[a] F. Bernsmann, Dr. J.-C. Voegel, Prof. V. Ball

Unité Mixte de Recherche 977

Institut National de la Santé et de la Recherche Médicale

11 rue Humann, 67085 Strasbourg Cedex (France)

Fax: (+33) 368853379

E-mail:vball@unistra.fr

[b] Dr. O. Ersen

Institut de Physique et Chimie des Matériaux

Unité Mixte de Recherche 7504

Centre National de la Recherche Scientifique

23 rue du Loess, 67087 Strasbourg (France)

[c] Dr. J.-C. Voegel, Prof. V. Ball

Université de Strasbourg, Faculté de Chirurgie Dentaire

1 Place de l'Hôpital, 67000 Strasbourg (France)

[d] E. Jan, Prof. N. A. Kotov

Department of Material Sciences and Engineering and

Department of Chemical Engineering

University of Michigan, Ann Arbor, Michigan 48109 (USA)

[e] Prof. V. Ball

Advanced Materials and Structures

Centre de Recherche Public Henri Tudor

L-4006 Esch-sur-Alzette (Luxembourg) 
produced by oxidation of dopamine in the absence of the adsorption substrate before being solubilised in alkaline solution.

Alkaline media promote deprotonation of carboxylic and phenolic groups carried by melanin ${ }^{[22]}$ and make it a polyanionic material. As a binding partner we chose PDADMAC, a cationic polyamine carrying quaternary ammonium groups, in order to promote electrostatic PDADMAC/dopamine-melanin interactions instead of covalent ones, which would occur with a polymer carrying primary or secondary amino groups. We investigated the morphology of the films produced by both methods as well as their permeability, and calculated their absorption coefficients. The optical and permeability data are consistent and demonstrate that the films produced by method 2 are less compact and more permeable than those produced by method 1, even though the film morphology seems similar in both cases. This investigation thus aims at demonstrating that the physicochemical properties of dopamine-melanin-containing films may be modulated over a broad range.

\section{Results and Discussion}

Dopamine solutions $\left(2 \mathrm{gL}^{-1}\right)$ in the presence of $50 \mathrm{~mm}$ Tris buffer at pH 8.5 were subjected to agitation ( $400 \mathrm{rpm}$ with a magnetic stirrer in a conical flask). After a few hours and in the absence of agitation, observation of sedimentation shows that micrometer-sized aggregates are forming. In a previous work we identified the reaction product as melanin by solid-state NMR spectroscopy. ${ }^{[10]}$ Aggregates form owing to the wellknown insolubility of melanin in weakly basic aqueous solutions. However, the solubility of melanin is markedly increased in accord with Bothma et al. ${ }^{[23]}$ by increasing the solution $\mathrm{pH}$ to 13 after $2 \mathrm{~h}$ of reaction at $\mathrm{pH} 8.5$. After $15 \mathrm{~min}$ of equilibration, the $\mathrm{pH}$ was decreased back to 12 . Such strongly basic conditions will of course induce some changes in the aggregation state of melanin. ${ }^{[24]}$ Solubilisation under basic conditions is most probably due to deprotonation of the phenolic and carboxylic moieties carried by the aggregates. ${ }^{[22]}$

To highlight the evolution of the aggregation state in solution as well as the slow consumption of dopamine, we took small aliquots from the reaction mixture at several intervals ranging from 0.5 to $166 \mathrm{~h}$ after titration of the solution to $\mathrm{pH}$ 12. Great care was taken to ensure a constant dilution (1/ 100 ) of the aliquots to compare the spectral properties of solutions having the same concentration (within 5-10\%). All UV/ Vis spectra are different from the spectrum of a $0.01 \mathrm{~g} \mathrm{~L}^{-1}$ dopamine solution at $\mathrm{pH} 5.9$, that is, under conditions in which dopamine is not oxidized (Figure 1).

The total concentration of aromatic groups was constant for all spectral measurements. After $30 \mathrm{~min}$ the dopamine-melanin solution already displays a black colour, which materialises in monotonic absorption at wavelengths above $500 \mathrm{~nm}$. The spectra contain two marked peaks close to 280 and $220 \mathrm{~nm}$, similar to the spectra of dopamine solutions, and an additional very broad peak around $440 \mathrm{~nm}$. This last spectral feature disappears rapidly (after about $2 \mathrm{~h}$ ). On the contrary, the intensity of the peak at $220 \mathrm{~nm}$ decreases only slowly and the peak at

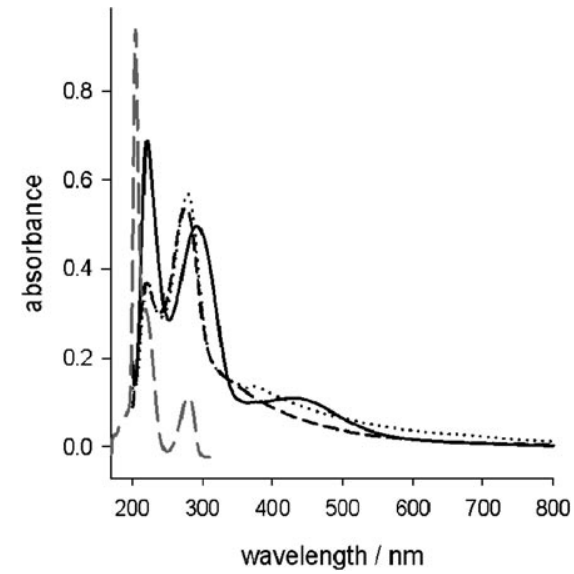

Figure 1. UV/Vis spectra of dopamine-melanin solutions. Dopamine $\left(2 \mathrm{gL}^{-1}\right)$ was allowed to react for $2 \mathrm{~h}$ in $50 \mathrm{~mm}$ Tris at $\mathrm{pH} \mathrm{8.5,} \mathrm{and} \mathrm{then} \mathrm{stored} \mathrm{for}$ $0.5 \mathrm{~h}$ (solid line), $24 \mathrm{~h}$ (dotted line) or $166 \mathrm{~h}$ (dashed line) at $\mathrm{pH} 12.0$ before measurement of the absorption spectrum. For spectrum acquisition, solutions were diluted $1 / 100$ in Tris buffer. The dashed grey line represents the spectrum of $0.01 \mathrm{~g} \mathrm{~L}^{-1}$ dopamine in $0.15 \mathrm{M} \mathrm{NaCl}$ at $\mathrm{pH} 5.9$.

$280 \mathrm{~nm}$ becomes a little stronger along with a slight blueshift for up to $24 \mathrm{~h}$ of reaction time. At the same time the absorption between 300 and $800 \mathrm{~nm}$ increases, which indicates progressive formation of dopamine-melanin. Longer reaction times only lead to very small changes in the spectra. Indeed, melanin is characterized by a broad band monotonic absorption profile. ${ }^{[15]}$ Although the composition of the solutions is probably very complex, our experiments show that even after long reaction times the transformation of dopamine to dopamine-melanin is by far not complete.

Preliminary dynamic light scattering experiments on dopamine-melanin solutions at $\mathrm{pH} 12$ (data not shown) were difficult to interpret owing to a badly defined intensity autocorrelation function at short timescales. Nevertheless, they confirmed a broad distribution of aggregate sizes with hydrodynamic radii up to a few micrometers. After filtration through a $0.2 \mu \mathrm{m}$ membrane, the obtained solution produced a lower scattering intensity but still contained micrometer-sized aggregates. This suggests that dynamic association-dissociation processes take place in the dopamine-melanin solutions. Similar dynamic phenomena were observed by Clancy and Simon in suspensions of natural Sepia officinalis melanin. ${ }^{[17]}$

To further examine the aggregation process, we deposited the particles contained in dopamine-melanin solutions in an LBL fashion with PDADMAC. More precisely we wanted to determine whether or not (PDADMAC/dopamine-melanin) films were rich in large grains. In the following experiments the employed dopamine-melanin solutions are allowed to react for $2 \mathrm{~h}$ at $\mathrm{pH} 8.5$ and kept for at least $24 \mathrm{~h}$ at $\mathrm{pH} 12$ before use, because the UV/Vis study presented above indicated that the solutions stabilised within one day after titration to $\mathrm{pH} 12$.

A quartz crystal microbalance with dissipation monitoring (QCM-D) of the build-up showed that a deposition time of $5 \mathrm{~min}$ is sufficient to reach steady-state deposition of both PDADMAC and dopamine-melanin (Figure $2 \mathrm{~A}$ ). Thus, a contact time of 5 min was used for all further experiments. It also ap- 
peared that the frequency changes measured at the third, fifth and seventh overtone of the quartz crystal overlapped, and this suggests that the obtained deposits are rigid, which was further confirmed by the small dissipation changes $\left(<10^{-5}\right.$,
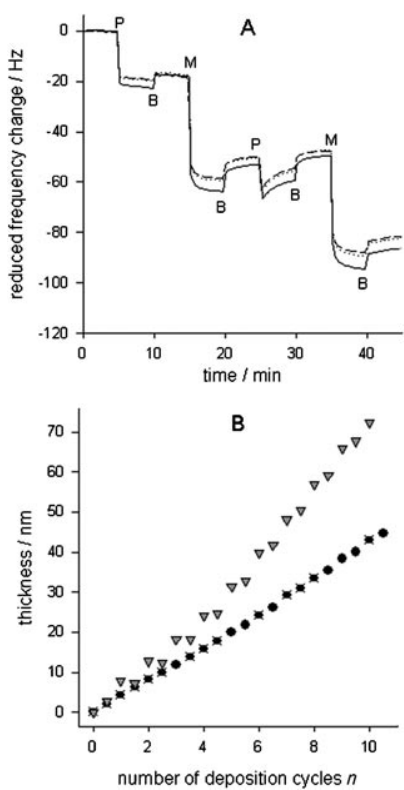

Figure 2. Deposition of (PDADMAC/dopamine-melanin) $n$ monitored by QCM-D. A) Reduced frequency changes at the 3rd (solid line), 5th (dotted line) and 7th (dashed line) overtone versus total deposition time. Letters in the graph indicate the injection of PDADMAC (P), water (B) or melanin solution (M) aged at $\mathrm{pH} 12$ for $5 \mathrm{~d}$ before the experiment. B) Thickness of deposits calculated from the reduced frequency change at the 3rd overtone by using the Sauerbrey equation ${ }^{[25]}$ versus number of deposition cycles. The employed melanin solution was aged at $\mathrm{pH} 12$ for 5 (triangles) or $6 \mathrm{~d}$ (circles).

data not shown). Hence, we used the Sauerbrey equation ${ }^{[25]}$ to convert the frequency changes to surface coverage and further to film thickness by using a specific mass of $1.2 \times 10^{3} \mathrm{~kg} \mathrm{~m}^{-3}$ for the (PDADMAC/dopamine-melanin) ${ }_{n}$ film (Figure $2 \mathrm{~B}$ ). It appears that the thickness grows linearly with the number of deposition cycles. The thickness increment is either equal for the deposition of PDADMAC and dopamine-melanin or greater for dopamine-melanin than for PDADMAC. At the present state of our investigation we do not know which factor decides between these two kinds of behaviour.

We also followed film deposition according to method 2 by ellipsometry on silicon slides (Figure 3) and UV/Vis spectroscopy on quartz slides (Figure 4). The thickness increment per layer pair of the films slightly increased with increasing ageing time at $\mathrm{pH} 12$ between dopamine-melanin preparation and the beginning of LBL deposition (inset in Figure 3). Hence, care must be taken to start the self-assembly between dopaminemelanin and PDADMAC at a given time after completion of the pigment's synthesis.

Figure 4 shows a linear increase of the UV/Vis absorbance of (PDADMAC/dopamine-melanin) $)_{n}$ deposits with increasing number of layer pairs $n$. The slope $k$ of the absorbance versus $n$ curve and the thickness increment per layer pair $d$ allow the

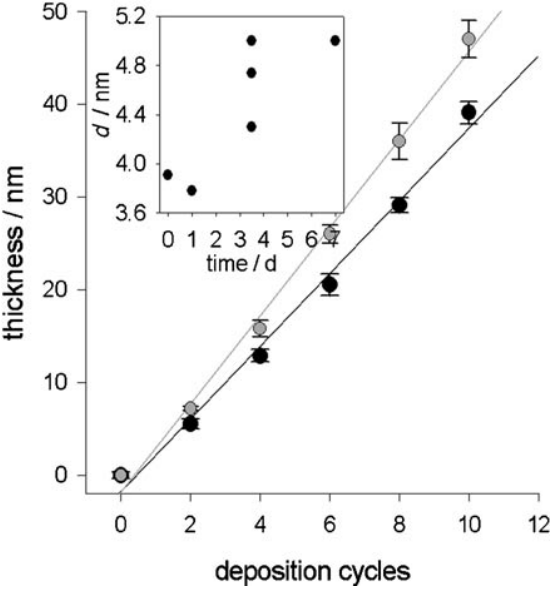

Figure 3. Thickness of (PDADMAC/dopamine-melanin) ${ }_{n}$ on silicon slides measured by ellipsometry (circles) and linear regressions to the data (lines). Error bars represent one standard deviation over five measurements on the same sample. Black circles: film made immediately after the end of the preparation of dopamine-melanin solution. Grey circles: film deposited after $3.5 \mathrm{~d}$ of ageing of the dopamine-melanin solution at $\mathrm{pH}$ 12. Inset: Thickness increment $d$ per layer pair as a function of ageing time of the melanin solution.

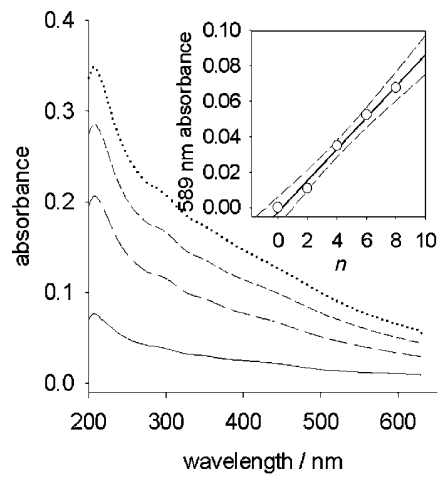

Figure 4. UV/Vis spectra of (PDADMAC/dopamine-melanin) films for $n=2$ (solid line), $n=4$ (long-dashed line), $n=6$ (short-dashed line) and $n=8$ (dotted line) deposited layer pairs. For these experiments, the dopaminemelanin solution was aged for $24 \mathrm{~h}$ before the deposition experiment. The inset displays the absorbance at $589 \mathrm{~nm}$ as a function of the number of deposited layer pairs. The straight line and the dashed lines correspond to a linear regression to the data and to the limits of the $95 \%$ confidence interval, respectively.

absorption coefficient $\varepsilon$ of the deposit at a given wavelength to be calculated [Eq. (1)]

$\varepsilon=k / 2 d$

where the coefficient of 2 accounts for the film coating on both sides of the quartz slide. We used dopamine-melanin solutions aged for $24 \mathrm{~h}$ for calculation of both $k$ and $d$. The value of $\varepsilon$ obtained for (PDADMAC/dopamine-melanin) $)_{n}$ films is $(1.2 \pm 0.3) \times 10^{6} \mathrm{~m}^{-1}$ at $589 \mathrm{~nm}$. It can be compared to the value obtained on dopamine-melanin films prepared by immersing substrates in multiple freshly prepared dopamine solutions (method 1). ${ }^{[10]}$ By substituting in Equation (1) $k$ with the 
absorbance and $d$ with the ellipsometric thickness of these films, we find $\varepsilon=(2.6 \pm 0.4) \times 10^{6} \mathrm{~m}^{-1}$ at $589 \mathrm{~nm}$. The fraction of melanin in the LBL (PDADMAC/dopamine-melanin) ${ }_{n}$ films can be estimated by comparison with dopamine-melanin films prepared according to method 1 to be about $50 \%$. This calculation relies on the fact that PDADMAC, the other component of the LBL films, is transparent at a wavelength of $589 \mathrm{~nm}$. Note, however, that films prepared by method 1 are not pure dopamine-melanin deposits but also contain large amounts of water. $^{[10]}$

The absorption spectrum of (PDADMAC/dopamine-melanin) $)_{n}$ films (Figure 4) is close to that of dopamine-melanin films deposited according to method $1 .{ }^{[10]}$ The absorbance decreases progressively over the entire UV/Vis spectrum and the marked UV peaks observed in the solution spectra (Figure 1) are not seen. This could mean that deposition from dopamine-melanin solutions is accompanied by a purification process with preferential deposition of the largest aggregates over dopamine or small oligomers. Another explanation for the strong decrease in the intensity of the UV absorption peaks during LBL deposition could be PDADMAC-induced aggregation of small molecules coming into contact with the final PDADMAC film.

To investigate the possibility of such a mechanism, we measured the UV/Vis spectrum of dopamine-melanin solutions diluted either in water at $\mathrm{pH} 12$ or in a $1 \mathrm{~g} \mathrm{~L}^{-1}$ PDADMAC solution at the same $\mathrm{pH}$ value (Figure 5). The two spectra are almost indistinguishable and close to that of a dopamine-mel-

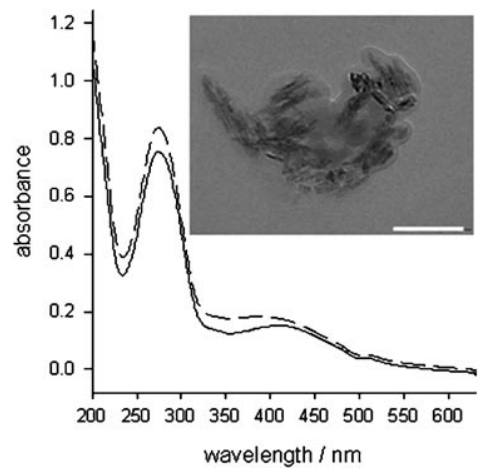

Figure 5. UV/Vis spectra of dopamine-melanin solutions prepared from $2 \mathrm{~g} \mathrm{~L}^{-1}$ dopamine solutions allowed to react for $2 \mathrm{~h}$ at $\mathrm{pH} 8.5$ and $15 \mathrm{~min}$ at $\mathrm{pH} 13.0$ before titration to $\mathrm{pH}$ 12.0. Then the solutions were immediately diluted by a factor of 100 in water (solid line) at $\mathrm{pH} 12$ or in a $1 \mathrm{~g} \mathrm{~L}^{-1}$ PDADMAC solution (dadshed line) at the same $\mathrm{pH}$. The inset is an electron micrograph of a typical dopamine-melanin aggregate from a solution immediately after $\mathrm{pH}$ adjustment to 12 . The scale bar corresponds to $100 \mathrm{~nm}$.

anin solution immediately after its synthesis, that is, they display an additional broad absorption band around $440 \mathrm{~nm}$ (as in Figure 1). Thus, contact of a dopamine-melanin solution with PDADMAC does not induce aggregation of melanin.

It is also interesting that the film topography (Figure 6) of films prepared by method 1 or 2 is similar in the sense that the sample surface is completely covered by grain-like structures. This is not surprising for films made by method 2, be-
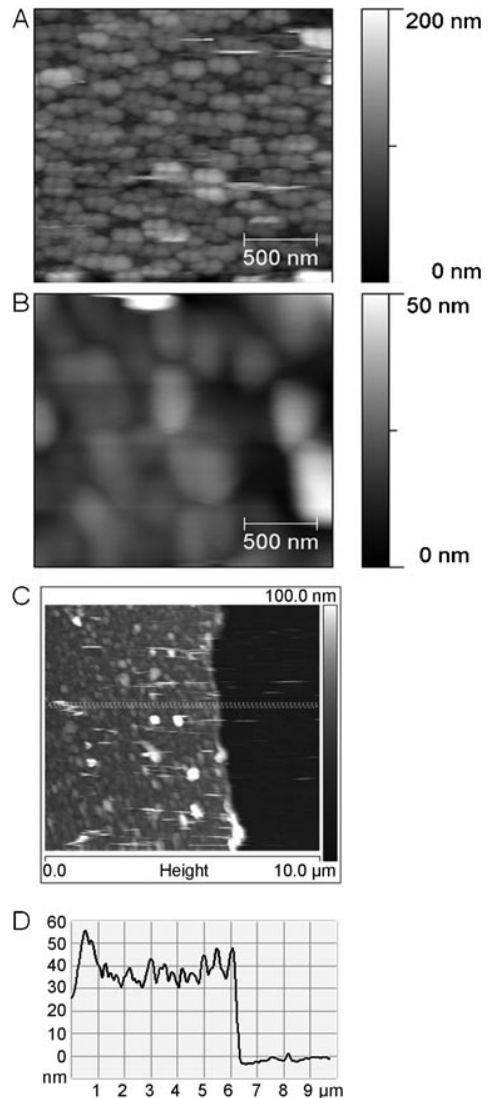

Figure 6. AFM topographies of dopamine-melanin films prepared by A) method 1 ( 85 immersions of $5 \mathrm{~min}$ in $2 \mathrm{gL}^{-1}$ dopamine solutions at $\mathrm{pH}$ 8.5) and $\mathrm{B})$ method 2 [(PDADMAC/dopamine-melanin $)_{10}$ film prepared using a melanin solution aged for $3 \mathrm{~d}$ at $\mathrm{pH}$ 12]. C) Needle-scratched (PDADMAC/ dopamine-melanin) ${ }_{10}$ film. D) Height profile of the dashed rectangle in (C).

cause these films are made from grains already present in solution. However films made according to method 1 are produced by successive dipping of the substrate in multiple freshly prepared dopamine solutions for only $5 \mathrm{~min}$. We previously showed that no dopamine-melanin film is formed when dopamine solutions are aged for $4 \mathrm{~h}$ at $\mathrm{pH} 8.5$ before immersion of the substrate. ${ }^{[10]}$ During 5 min of oxidation formation of dopamine-melanin in solution is by far not complete and no large particles are present. This finding strongly suggests that the particles found in Figure $6 \mathrm{~A}$ originate from a surface-induced aggregation process.

Careful comparison between AFM images of films made according to method 1 (Figure 6A) and method 2 (Figure 6B) suggests that (PDADMAC/dopamine-melanin) ${ }_{10}$ films are made of larger grains that lead to a less compact structure than for films made by 85 successive immersions in freshly prepared dopamine solutions. The finding of a less compact arrangement of particles for films prepared according to method 2 is in agreement with their lower absorption coefficient compared with those prepared according to method 1. In the case of (PDADMAC/dopamine-melanin) ${ }_{10}$ films, the thickness determined by AFM of about $40 \mathrm{~nm}$ is similar to that calculated from ellipsometry measurements (Figure 3 ) and justifies the use of a fixed refractive index of 1.465 for evaluation of the el- 
lipsometry data. In addition, the thickness increment per layer pair (about $4.5 \mathrm{~nm}$, Figure 3) is much smaller than the apparent size of the particles present in solution ( $>200 \mathrm{~nm}$, inset of Figure 5) and on the surface (>100 nm, Figure 6). To explain this difference, we assume that the dopamine-melanin particles have a highly anisotropic shape and are deposited with their largest dimension parallel to the surface.

We wanted to measure whether a change in dopamine-melanin particle packing density translates into a change in film permeability. To that end, we performed impedance spectroscopy and cyclic voltammetry (CV) in the presence of $1 \mathrm{~mm}$ ferrocynanide anions. The dopamine-melanin films produced according to method 1 were almost impermeable to ferrocyanide after 12 successive immersion steps in freshly prepared dopamine solutions leading to a film thickness of $7 \mathrm{~nm} .^{[14]}$

A dopamine-melanin film made by 12 successive immersions in dopamine solutions displays a much higher impedance modulus $\left(Z^{\prime 2}+Z^{\prime 2}\right)^{1 / 2}$ than (PDADMAC/dopamine-melanin) ${ }_{10}$ films (Figure 7), although the later films, with thickness of $40 \mathrm{~nm}$, are almost six times as thick as the former (Figure 3).

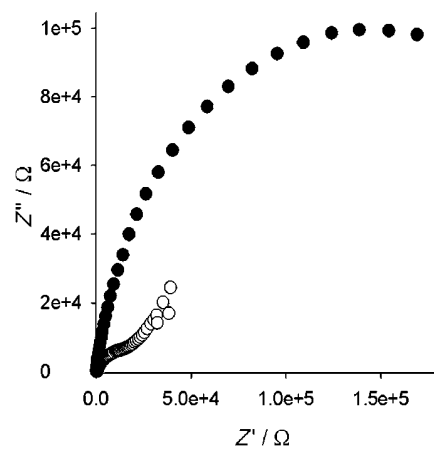

Figure 7. Impedance spectra (opposite of the imaginary part $-Z^{\prime \prime}$ vs real part $Z^{\prime}$ of the impedance measured at frequencies from $10^{5}$ to $10^{-2} \mathrm{~Hz}$ ) of dopamine-melanin films made by 12 successive immersions of $5 \mathrm{~min}$ in dopamine solutions (method 1, filled circles) and of (PDADMAC/dopaminemelanin) ${ }_{10}$ films (empty circles). The dopamine-melanin was aged $24 \mathrm{~h}$ before deposition of (PDADMAC/dopamine-melanin) ${ }_{10}$ films. The measurements were performed in the presence of $10 \mathrm{mM}$ Tris buffer $(\mathrm{pH} 7.5)+150 \mathrm{~mm} \mathrm{NaNO}{ }_{3}+1 \mathrm{~mm} \mathrm{~K}_{4}\left[\mathrm{Fe}(\mathrm{CN})_{6}\right]$.

Indeed, for films homogeneous in composition one expects a linear increase of the impedance modulus with increasing film thickness. The films prepared according to an LBL deposition method display also a clear linear branch in the low-frequency domain, which reflects the importance of mass transfer for the impedance (Warburg impedance).

In addition, the LBL films display some permeability to ferrocyanide anions, whereas the films prepared by method 1 are totally impermeable. Indeed their cyclic voltammograms (CV) are purely capacitive curves (Figure 8 ). The decrease of the oxidation-reduction current for films produced according to method 1 may be due to very low film porosity and/or to the fact that the dopamine-melanin films are negatively charged at the $\mathrm{pH}$ of the experiment. ${ }^{[14]}$ The larger oxidation-reduction currents for (PDADMAC/dopamine melanin) ${ }_{10}$ films may be due to increased porosity and/or to the presence of the posi-

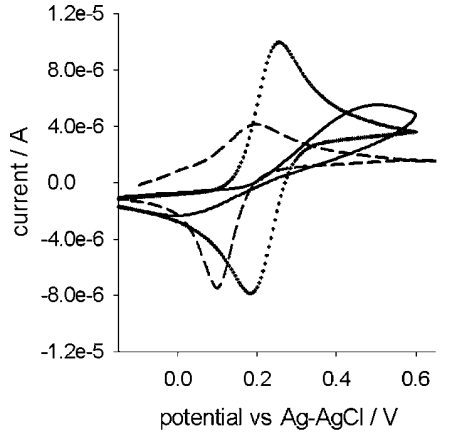

Figure 8. Cyclic voltammetry curves of dopamine-melanin films made by 12 successive immersions of $5 \mathrm{~min}$ in dopamine solutions (method 1, solid line), of (PDADMAC/dopamine-melanin) ${ }_{10}$ films (dashed line) and of the pristine amorphous carbon electrode (crosses). The dopamine-melanin was aged for $24 \mathrm{~h}$ before deposition of (PDADMAC/dopamine-melanin) ${ }_{10}$ films. The measurements were performed in the presence of $10 \mathrm{~mm}$ Tris buffer $(\mathrm{pH} 7.5)+150 \mathrm{~mm} \mathrm{NaNO}_{3}+1 \mathrm{~mm} \mathrm{~K}_{4}\left[\mathrm{Fe}(\mathrm{CN})_{6}\right]$.

tively charged PDADMAC chains, which are traps for ferrocyanide anions.

The presence of a (PDADMAC/dopamine-melanine $)_{10}$ film on the surface of an amorphous carbon electrode reduces the maximal anodic current corresponding to the oxidation of ferrocyanide $\left[\mathrm{Fe}(\mathrm{CN})_{6}\right]^{4-}$ to ferricyanide $\left[\mathrm{Fe}(\mathrm{CN})_{6}\right]^{3-}$ anions by about $50 \%$ (Figure 8 ). On the other hand, the reduction current is hardly affected. This may be due to preferential binding to the film of $\left[\mathrm{Fe}(\mathrm{CN})_{6}\right]^{3-}$ compared to $\left[\mathrm{Fe}(\mathrm{CN})_{6}\right]^{4-}$. This explanation is plausible owing to the finding that both the oxidation and reduction peaks are shifted to less positive values in the presence of the film. The peak separation of $101 \mathrm{mV}$ is much higher than on the pristine electrode $(70 \mathrm{mV})$, so that the oxidation-reduction process is not reversible in presence of the (PDADMAC/dopamine-melanin) ${ }_{10}$ film. In addition, the apparent redox potential of the $\left[\mathrm{Fe}(\mathrm{CN})_{6}\right]^{3-} /\left[\mathrm{Fe}(\mathrm{CN})_{6}\right]^{4-}$ couple, calculated as the average of the potentials at the peak positions, is $150 \mathrm{mV}$ in the presence of the film but $220 \mathrm{mV}$ on the pristine electrode, which suggests that the film has a Donnan potential of $-70 \mathrm{mV}$. This indeed indicates that the trivalent ferricyanide anion binds preferentially compared to the tetravalent ferrocyanide anion. In a forthcoming study we will investigate the $\mathrm{CV}$ of other redox probes on (PDADMAC/dopamine-melanin) films (method 2) as well as on dopamine-melanin films from method 1.

\section{Conclusions}

We have demonstrated that dopamine-melanin films made by successive immersion of a substrate in freshly prepared dopamine solutions $\left(2 \mathrm{gL}^{-1}\right.$ in presence of $50 \mathrm{~mm}$ Tris buffer at $\mathrm{pH}$ 8.5, method 1) and LBL films made by successive immersions in PDADMAC and dopamine-melanin solutions (method 2) lead to deposits of controllable thickness. The deposits consist of anisotropic grains with a maximal dimension in the size range of hundreds of nanometers. Such grains are present in the solutions used for LBL deposition but not in those used for deposition according to method 1, which sug- 
gests that they self-assemble at the substrate-solution interface. Our investigation shows that by using LBL deposition from solutions containing dopamine-melanin, one can deposit films rich in this fascinating biomolecule but having properties distinctively different from the films produced directly by oxidation of dopamine. Notably the permeability of the LBL films to ferrocyanide ions is higher, which might be important for applications involving permselective coatings, for example fuel-cell separation membranes. ${ }^{[26]}$

\section{Experimental Section}

Dopamine (ref. H8502) and PDADMAC (ref. 409014, 20 wt \% solution in water) were purchased from Sigma-Aldrich (Saint Louis, Missouri, USA) and used without further purification. All solutions were made from doubly deionised water (Milli Q Plus system, Millipore, Billerica, Massachusetts, USA) with a resistivity of $182 \mathrm{k} \Omega \mathrm{m}$. The buffer solution used for dopamine-melanin synthesis was $50 \mathrm{~mm}$ Tris (Tris(hydroxymethylamino)methane, Sigma-Aldrich, ref. T1503) at $\mathrm{pH}$ 8.5. The buffer solution used for the electrochemical characterizations was $10 \mathrm{~mm}$ Tris at $\mathrm{pH} 7.5$ with $150 \mathrm{~mm} \mathrm{NaNO}_{3}$ (Sigma-Aldrich, ref. S5506). For the permeability and impedance spectroscopy measurements, potassium ferrocyanide trihydrate (Sigma-Aldrich, ref. P9387) was dissolved at $1 \mathrm{~mm}$ in the buffer solution containing Tris and $\mathrm{NaNO}_{3}$. The PDADMAC solutions were dissolved at $1 \mathrm{gL}^{-1}$ in $50 \mathrm{~mm}$ Tris buffer and their $\mathrm{pH}$ was adjusted to 12 before LBL deposition. The $\mathrm{pH}$ of all solutions was measured with a HI8417 pH meter (Hanna Instruments, Tanneries, France) and adjusted by addition of concentrated hydrochloric acid (Merck Darmstadt, Germany, ref. 1.01834.2500) or sodium hydroxide solution (Sigma-Aldrich, ref. S-5881).

Film Deposition According to Method 1: Deposition of melanin films from dopamine solutions at $2 \mathrm{~g} \mathrm{~L}^{-1}$ in the presence of $50 \mathrm{~mm}$ Tris buffer at pH 8.5 and naturally dissolved oxygen has been described in detail elsewhere. ${ }^{[10]}$ The most important points are that dopamine must be dissolved in the buffer just before being brought into contact with the substrate for $5 \mathrm{~min}$, that this operation must be repeated $m$ times (mostly $m=12$ in this investigation) and that the films are neither rinsed with buffer nor dried between two successive immersion steps in dopamine solutions.

Film Deposition According to Method 2: To prepare (PDADMAC/ dopamine-melanin) ${ }_{n}$ films, the substrates were immersed successively in a PDADMAC solution for $t$ min, rinsed with water (without $\mathrm{pH}$ adjustment), immersed in a dopamine-melanin solution (initial dopamine concentration: $2 \mathrm{~g} \mathrm{~L}^{-1}$ ) for $t$ min and finally rinsed with water. The optimal duration of the deposition steps was determined from the quartz crystal microbalance (QCM) experiments to obtain steady-state adsorbed amounts. These four steps were repeated $n$ times to obtain a film denoted (PDADMAC/dopaminemelanin). The dopamine-melanin solutions were prepared from dopamine solutions, agitated with a magnetic stirrer at $400 \mathrm{rpm}$ for $2 \mathrm{~h}$ in contact with ambient air. The solution was then titrated to $\mathrm{pH} 13.0$ with concentrated $\mathrm{NaOH}$ solution to optimise the solubility of the dopamine-melanin aggregates. Finally, after $15 \mathrm{~min}$ of equilibration the dopamine-melanin solution was titrated back to $\mathrm{pH}$ 12.0. For spectroscopic characterization, the dopamine-melanin solutions were diluted $1 / 100$ either in Tris buffer $(50 \mathrm{~mm}, \mathrm{pH} 12.0)$ or in the same buffer containing $1 \mathrm{~g} \mathrm{~L}^{-1}$ PDADMAC.

Adsorption Substrates: The films used for spectroscopic characterization were deposited on quartz slides (Thuet, Blodesheim, France), and silicon slides (Siltronix, Archamps, France) were used for film-thickness measurements by ellipsometry. Both substrates were cleaned by immersion in a freshly prepared piranha solution [two parts of $98 \% \mathrm{H}_{2} \mathrm{SO}_{4}$ and one part of $30 \% \mathrm{H}_{2} \mathrm{O}_{2}$ ) for $30 \mathrm{~min}$ and intensively rinsed with distilled water (caution! Piranha solution is highly corrosive and reacts violently with organic matter]. This cleaning was performed just before film deposition. Silica coated-quartz cristals (ref. QSX 303, Q-Sense, Göteborg, Sweden) were used to follow film deposition by means of QCM-D. The crystals were cleaned in situ in the QCM-D measurement chamber by successive contact with $2 \%$ Hellmanex (Hellma GmbH, Müllheim, Germany) solution for $30 \mathrm{~min}$, water, $0.1 \mathrm{~m} \mathrm{HCl}$ solution (10 min) and water once more. The substrates coated with dopamine-melanin films or with (PDADMAC/dopamine-melanin) ${ }_{n}$ films were removed from the QCM chamber, dried under a stream of nitrogen and used for AFM imaging. For cyclic voltammetry (CV) experiments, amorphous carbon electrodes ( $\mathrm{CH}$ Instruments, Austin, Texas, USA, ref. 104) served as substrates for film deposition. The electrodes were polished on $0.05 \mu \mathrm{m}$ diameter $\gamma$ alumina powder (Buehler, Lake Bluff, Illinois, USA, ref. 40-6325-008) in three successive steps of 2 min separated by rinsing with water. Then the electrodes were sonicated at $35 \mathrm{kHz}$ for $2 \times 3 \mathrm{~min}$ in a Transonic TI-H-50 sonicator (Laval Lab, Laval, Canada).

Characterization Techniques: UV/Vis spectra were acquired with an $\mathrm{mc}^{2}$ spectrophotometer (SAFAS, Monaco) between 200 and $630 \mathrm{~nm}$ with a spectral resolution of $1 \mathrm{~nm}$. In the case of melanincontaining films, the absorbance was calculated by using the transmission across the cleaned quartz slide as reference. The absorption spectra of the diluted dopamine-melanin solutions were acquired in quartz cuvettes with $1 \mathrm{~cm}$ path length in double-beam mode with a reference cuvette filled with Tris buffer adjusted to $\mathrm{pH} 12$.

Quartz crystal microbalance with dissipation (QCM-D, Q-Sense 300, Göteborg, Sweden) experiments were performed to investigate the deposition kinetics of dopamine-melanin films (data given in ref. [10]) and of (PDADMAC/dopamine-melanin) films. The silicacoated quartz crystal was put in a measurement chamber $(100 \mu \mathrm{L}$ in volume), cleaned in situ, and the resonance frequency of the quartz crystal was followed as function of time at the fundamental frequency (close to $5 \mathrm{MHz}$ ) as well as at its 3rd, 5th and 7th overtones. In case where the reduced frequency changes, $\Delta F_{i} / i$ (where $i$ is the overtone number, $i=1,3,5,7)$ overlap, the film deposited at the quartz crystal/solution interface can be considered to be rigid, and under these conditions, the Sauerbrey equation [Eq. (2)] can be used to calculate the surface coverage $\Gamma$ from $\Delta F_{i} / i$ according to:

$\Gamma=-C\left(\Delta F_{i}\right) / i$

The value of the proportionality constant $C$ depends on the quartz density and on its shear modulus and is $16.6 \mathrm{ng} \mathrm{cm}^{-2} \mathrm{~Hz}^{-1}$ for the employed QSX 303 crystals. From the surface coverage, the film thickness $d$ can be calculated provided the density $\rho$ of the deposited film is known [Eq. (3)].

$d=\frac{\Gamma}{\rho}$

The density of dopamine-melanin was determined in our previous investigation ${ }^{[10]}$ to be $1.2 \mathrm{~kg} \mathrm{~m}^{-3}$, a value which is close to that of most polymers. As the (PDADMAC/dopamine-melanin) ${ }_{n}$ films are made from PDADMAC, dopamine-melanin and electrolyte solution, their density should lie between 1.0 and $1.2 \mathrm{~kg} \mathrm{~m}^{-3}$. We chose a value of $1.2 \mathrm{~kg} \mathrm{~m}^{-3}$ in our calculations, which could induce a slight 
underestimation of the film thickness. On the other hand, the calculated value of $\Gamma$ takes the water of hydration of the films into account, which may lead to an overestimation of the film thickness.

Dopamine-melanin-containing films built on cover glasses were dried under a stream of nitrogen and imaged by atomic force microscopy (AFM). Topography images in contact mode were acquired with a Nanoscope IV instrument (Veeco, Santa Barbara, California, USA) at a scanning frequency of $2 \mathrm{~Hz}$. The employed cantilevers (Veeco, ref. MSCT-AUWH) have a nominative spring constant of $0.01 \mathrm{~N} \mathrm{~m}^{-1}$ and are terminated with a silicon nitride tip having a nominative radius of curvature of $10 \mathrm{~nm}$. To validate the dopamine-melanin thickness calculated from ellipsometry and QCM-D experiments, the sample was scratched with a syringe needle previously cleaned with ethanol, and the height difference between the scratched and the unscratched area was used as a measure for the thickness of the deposit. Further surface topography images of the dopamine-melanin films produced according to method 1 can be found in our previous publications. ${ }^{[10,14]}$

Ellipsometry (Horiba Jobin Yvon model PZ2000, Longjumeau, France) was used to measure the thickness evolution of (PDAD-

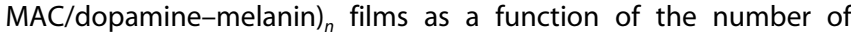
layer pairs $n$ and as a function of the ageing time of dopaminemelanin solutions between the end of their preparation and the beginning of LBL film deposition. The measurements were performed at a constant wavelength $(\lambda=632.8 \mathrm{~nm})$ and at a constant angle of incidence $\left(70^{\circ}\right)$. The films were dried under a stream of nitrogen before the measurement and rehydrated with distilled water before continuation of film deposition. Some experiments were performed up to the deposition of ten layer pairs without intermediate drying, and the calculated film thickness was compared to that of films made from the same number of layer pairs but with five intermediate drying steps. No significant difference was found, that is, the intermediate drying-rehydration steps did not influence film deposition. Each thickness value represents the average over five measurements along the main axis of the used silicon wafers. We assumed the deposit to be homogeneous and isotropic and to have a refractive index of 1.465 (at $\lambda=632.8 \mathrm{~nm}$ ).

Cyclic voltammetry $(\mathrm{CV})$ and impedance spectroscopy were performed with a conventional three-electrode set-up using a $\mathrm{CHI}$ $604 \mathrm{~B}$ potentiostat ( $\mathrm{CH}$ Instruments). The reference and auxiliary electrode were an $\mathrm{Ag} / \mathrm{AgCl}$ electrode (ref. 111) and a platinum wire (ref. 115) respectively. The CV curves were measured by cycling the potential ( $\mathrm{vs} \mathrm{Ag} / \mathrm{AgCl}$ ) between $-0.10 \mathrm{~V}$ and $0.70 \mathrm{~V}$ at a scanning rate of $50 \mathrm{mV} \mathrm{s}^{-1}$. The mean value of the oxidation peak potential and the reduction peak potential defines the apparent redox potential. The difference between the redox potentials of the pristine electrode and the electrode covered with the considered film gives the Donnan potential of the film. Electrochemical impedance spectroscopy (EIS) was performed on dopamine-melanin films made by 12 successive immersions in freshly prepared dopamine solutions ( 5 min for each contact) or on (PDADMAC/dopamine-melanin) ${ }_{10}$. The dc potential was set at $220 \mathrm{mV}$ versus $\mathrm{Ag} / \mathrm{AgCl}$, that is, at the apparent redox potential of the $\left[\mathrm{Fe}(\mathrm{CN})_{6}\right]^{3-} /\left[\mathrm{Fe}(\mathrm{CN})_{6}\right]^{4-}$ couple, and the amplitude of the ac potential was $5 \mathrm{mV}$. The frequency was decreased from $10^{5}$ to $10^{-2} \mathrm{~Hz}$, and 12 measurements were performed per frequency decade.

Transmission Electron Microscopy: The TEM experiments were performed on a TOPCON 002 electron microscope operating at
$200 \mathrm{kV}$, with $0.18 \mathrm{~nm}$ point-to-point resolution. By using the conventional parallel TEM mode, several bright-field images were acquired on the studied specimens with $100 \mu \mathrm{m}$ objective aperture diameter to enhance the contrast. No visible irradiation damage was observed on the specimens after the acquisition process.

\section{Acknowledgements}

N.A. Kotov thanks the Gutenberg Foundation for financial support. F. Bernsmann acknowledges financial support by the Alsace region.

Keywords: biopolymers - layer-by-layer deposition - materials science $\cdot$ melanin $\cdot$ thin films

[1] T. Sun, L. Feng, X. Gao, L. Jiang, Acc. Chem. Res. 2005, 38, 644-652.

[2] C. Dorrer, J. Rühe, Soft Matter 2009, 5, 51-61.

[3] A. Abbas, C. Vivien, B. Bocquet, D. Guillochon, P. Supiot, Plasma Processes Polym. 2009, 6, 593-604.

[4] K. Ariga, J. P. Hill, Q. Ji, Chem. Phys. Phys. Chem. 2007, 9, 2319-2340.

[5] Y. Wang, A. S. Angelatos, F. Caruso, Chem. Mater. 2008, 20, 858-858.

[6] G. Decher, J. D. Hong, Ber. Bunsen-Ges. 1991, 95, 1430-1434.

[7] Y. Shimazaki, R. Nakamura, S. Ito, M. Yamamoto, Langmuir 2001, 17, 953-956.

[8] W. B. Stockton, M. F. Rubner, Macromolecules 1997, 30, 2717-2725.

[9] H. Lee, S. M. Dellatore, W. M. Miller, P. B. Messersmith, Science 2007, 318, $426-430$.

[10] F. Bernsmann, A. Ponche, C. Ringwald, J. Hemmerle, J. Raya, B. Bechinger, J. C. Voegel, P. Schaaf, V. Ball, J. Phys. Chem. C 2009, 113, 8234-8242.

[11] S. M. Kang, J. Rho, I. S. Choi, P. B. Messersmith, H. Lee, J. Am. Chem. Soc. 2009, 131, 13224-13225.

[12] C. J. Bettinger, P. P. Bruggeman, A. Misra, J. T. Borenstein, R. Langer, Biomaterials 2009, 30, 3050-3057.

[13] H. Lee, J. Rho, P. B. Messersmith, Adv. Mater. 2009, 21, $431-434$.

[14] F. Bernsmann, B. Frisch, C. Ringwald, V. Ball, J. Colloid Interface Sci. 2010, 344, 54-60.

[15] P. Meredith, B. J. Powell, J. Riesz, S. P. Nighswander-Rempel, M. R. Pederson, E. G. Moore, Soft Matter 2006, 2, 37-44.

[16] M. d'Ischia, A. Napolitano, A. Pezzella, P. Meredith, T. Sarna, Angew. Chem. 2009, 121, 3972-3979; Angew. Chem. Int. Ed. 2009, 48, $3914-$ 3921.

[17] C. M. R. Clancy, J. D. Simon, Biochemistry 2001, 40, 13353-13360.

[18] J. Riesz, J. Gilmore, P. Meredith, Biophys. J. 2006, 90, 4137-4144.

[19] A. Pezzella, A. ladonisi, S. Valerio, L. Panzella, A. Napolitano, M. Adinolfi, M. d'Ischia, J. Am. Chem. Soc. 2009, 131, 15270-15275.

[20] M. Abbas, F. D'Amico, L. Morresi, N. Pinto, M. Ficcadenti, R. Natali, L. Ottaviano, M. Passacantando, M. Cuccioloni, M. Angeletti, R. Gunnella, Eur. Phys. J. E 2009, 28, 285-291.

[21] M. I. N. da Silva, S. N. Dezidério, J. C. Gonzalez, C. F. O. Graeff, M. N. Cotta, J. Appl. Phys. 2004, 96, 5803-5807.

[22] S. Ito, Biochim. Biophys. Acta Gen. Subj. 1986, 883, 155-161.

[23] J. P. Bothma, J. de Boor, U. Divakar, P. E. Schwenn, P. Meredith, Adv. Mater. 2008, 20, 3539-3542.

[24] M. G. Bridelli, Biophys. Chem. 1998, 73, 227-239.

[25] G. Sauerbrey, Z. Phys. 1959, 155, 206-222.

[26] J. Wang, L. Xiao, Y. Zhao, H. Wu, Z. Jiang, W. Hou, J. Power Sources 2009, 192, 336-343.

Received: May 14, 2010

Published online on August 30, 2010 